\title{
Intake ground vortex characteristics
}

\author{
Nityabh Mishra, David G MacManus and John Murphy \\ Cranfield University, UK
}

\begin{abstract}
The development of ground vortices when an intake operates in close proximity to the ground has been studied computationally for several configurations including front and rear quarter approaching flows as well as tailwind arrangements. The investigations have been conducted at model scale using a generic intake geometry. Reynolds Averaged Navier-Stokes calculations have been used and an initial validation of the computational model has been carried out against experimental data. The computational method has subsequently beenapplied to configurations that are difficult to test experimentally including tailwind and rear quarter flows. The results, along with those from a previous compatible study of headwind and pure crosswind configurations, have been used to assess the ground vortex behaviour under a broad range of velocity ratios and approaching wind angles. The characteristics provide insights on the influence of the size and strength of ground vortices on the overall quality of the flow ingested by the intake.
\end{abstract}

\section{Keywords}

Intake, ground vortex, RANS, headwind, crosswind, tailwind,distortion

Corresponding Author: Dr David G MacManus, Department of Power \& Propulsion, Cranfield University, Cranfield MK43 0AL, UK. Email: D.G.Macmanus@cranfield.ac.uk

\section{Nomenclature}

\section{English Symbols}
D
Diameter $[\mathrm{m}]$
$\mathrm{H} \quad$ Intake centre-line height from ground plane [m]
$\mathrm{h}$ Vertical distance of lowest point on highlight plane to the ground [m]
$\mathrm{P}_{0} \quad$ Total pressure $[\mathrm{Pa}]$
q
Dynamic pressure $[\mathrm{Pa}]$ 


$\begin{array}{ll}\mathrm{U} & \text { Velocity magnitude }[\mathrm{m} / \mathrm{s}] \\ \mathrm{u}, \mathrm{v}, \mathrm{w} & \text { Cartesian velocity components }[\mathrm{m} / \mathrm{s}] \\ \mathrm{x}, \mathrm{y}, \mathrm{z} & \text { Cartesian coordinates }[\mathrm{m}] \\ \mathrm{y}^{+} & \text {Non-dimensional wall distance }[-]\end{array}$

\section{Greek Symbols}

\begin{tabular}{ll}
$\Gamma$ & Circulation $\left[\mathrm{m}^{2} / \mathrm{s}\right]$ \\
$\delta^{*}$ & Boundary layer momentum thickness $[\mathrm{m}]$ \\
\hline & Density $\left[\mathrm{kg} / \mathrm{m}^{3}\right]$ \\
Subscripts & Yaw angle $\left[^{\circ}\right]$ \\
crit & Vortex blow-away condition \\
$\mathrm{f}$ & Fan face \\
$\mathrm{i}$ & Intake duct \\
$\mathrm{I}$ & Highlight plane \\
$\mathrm{x}, \mathrm{y}, \mathrm{z}$ & Components in Cartesian coordinates \\
$\mathrm{r}, \theta, \mathrm{ax}$ & Components in cylindrical coordinates \\
$\propto$ & Freestream conditions
\end{tabular}

\section{Non-Dimensionals}

$\Gamma^{*} \quad$ Average non-dimensional vortex strength $\left(\Gamma / \mathrm{U}_{i} \mathrm{D}_{1}\right)$

$\mathrm{U}^{*} \quad$ Ratio of intake velocity to freestream velocity, $\mathrm{U}_{\mathrm{i}} / \mathrm{U}_{\infty}$

$\eta \quad$ Normalised non-dimensional vortex strength

DC(60) Distortion coefficient

SC(60) Swirl distortion coefficient

$\zeta \quad$ Total pressure loss coefficient

M Mach number

\section{Introduction}

When an aircraft engine operates in close proximity to the ground, under static or near-static conditions, a strong vortex is formed at the ground plane which is ingested into the intake. This vortex, referred to as a 'ground vortex', can develop under headwind, crosswind, tailwind, 


\section{Accepted manuscript}

quiescent and intermediate yaw angle conditions [1-11]. The ingestion of such a vortex has been reported to have an adverse effect on engine performance and integrity. In particular, the vortex can cause foreign object damage $[8,12]$, compressor surge $[13,14]$, fan vibration $[15,16]$, and massflow distortion resulting in premature lip separation [4].

For a ground vortex to form the intake sucked streamtube must interact with the ground plane and there must be a source of vorticity in the intake flow-field. [10]. In general, the latter is always satisfied if the former condition is fore filled. The relative strength of ground vortices is dependent on the degree of interaction between the intake sucked streamtube and the ground plane $[2,6,17]$. This interaction is primarily dependent on the intake non-dimensional ground clearance $\left(h / D_{l}\right)$ and the streamtube contraction ratio of the intake $\left(U_{i} / \bigcup_{\infty}\right.$ for an incompressible flow). Based on these two non-dimensional parameters, a number of empirical correlations have been reported to identify the vortex formation limit $[5,11,17,18]$. Previous studies have shown that there are three primary sources of vorticity $[9,10]$ - the suction induced [9], and approaching freestream boundary layers [10], and the flow over the external intake surface [10]. The relative dominance of these vorticity sources strongly influences the vortex form and strength and is primarily dependent on the approaching wind angle $(\psi)$ and the velocity ratio $\left(U^{*}\right)[1,6,9,10]$. Brix found that under quiescent conditions $\left(U^{*}=\infty\right)$, the flow-field is dominated by vorticity associated with the suction induced boundary layer [9]. The concentration and amplification of this vorticity causes two relatively weak contra-rotating vortices to be ingested. The vortex topology is such that the right hand vortex rotates clockwise when facing the intake highlight plane (Figure 1a).

Under headwind conditions, as the velocity ratio is reduced by increasing $U_{\infty}$, the vorticity within the approaching boundary layer increases. At a particular headwind velocity ratio, the suction induced and approaching boundary layer vorticity sources become equal in magnitude. Brix identified this transition phase and observed the appearance of two pairs of highly unsteady contra-rotating vortices [9]. With further reductions in the velocity ratio, vorticity within the approaching boundary layer begins to dominate the flowfield and once again a single pair of contra-rotating vortices is observed. These vortices are stronger and rotate with an opposite 
sense compared to those formed under quiescent conditions (Figure 1b). Computational studies by Zantopp found that the vortices generated under headwind conditions are unsteady and that the flow oscillates between a range of states including vortex pairs as well as modulating single vortex modes [6]. Reducing the velocity ratio leads to an increase in the strength of the vortices until a peak value is reached [5]. A further decrease in velocity ratio causes a reduction in vortex strength due to the reduced interaction between the sucked streamtube and the ground. Eventually, a critical velocity ratio $\left(U^{*}\right.$ crit $)$ is reached where the sucked streamtube no longer interacts with the ground and the ground vortex does not form $[5,6]$.

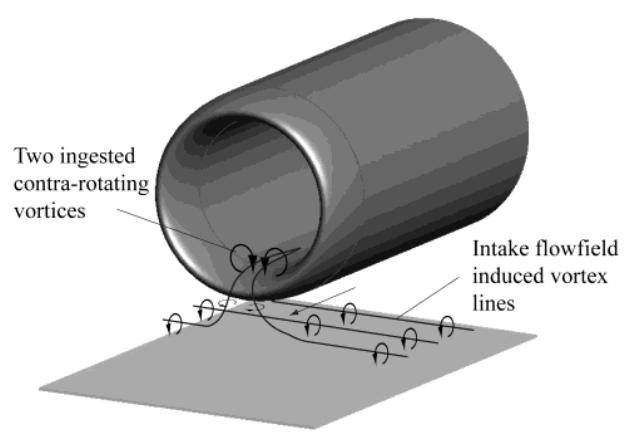

(a)

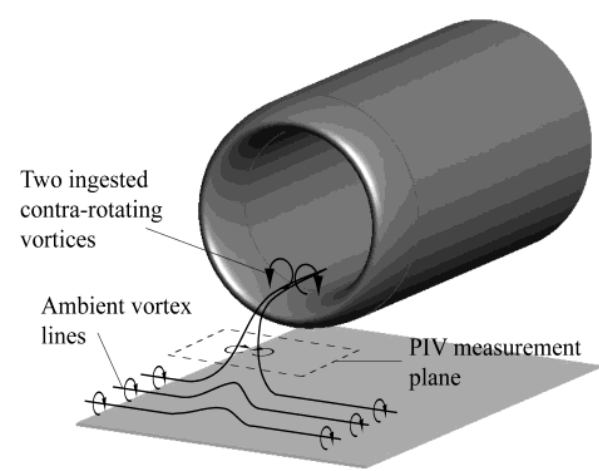

(b)

Figure 1: Basic ground vortex flow topologies under (a) no-wind (b) headwind.

Under crosswind conditions $\left(\psi=90^{\circ}\right)$ the ground vortex exhibits a different formation mechanism. Vorticity from the ground boundary layers has a less significant impact and vorticity from the external intake surface now dominates [10]. The flow field typically comprises a single ground vortex that is relatively steady in both strength and position and is considerably stronger than the vortices generated under quiescent and headwind conditions [4]. This single ground vortex is accompanied by a 'trailing vortex' which is also ingested by the intake and arises on the leeward side. Until recently, these trailing vortices were believed to emanate on the leeward side of the intake and to continue downstream [10]. However, Zantopp et al proposed an alternative flow topology [6]. As postulated by de Sievri et al the trailing vortex is a result of vorticity being generated over the external surface of the intake [10]. However, once this vorticity reaches the edge of the sucked streamtube, it can no longer propagate downstream. Figure 2 shows the calculated streamlines which are drawn back towards the intake and form the core of the ingested trailing vortex. An additional trailing vortex also forms downstream with the main vorticity source once again associated with the intake external surface. This vortex is 


\section{Accepted manuscript}

outside of the sucked streamtube and it is not ingested by the intake (Figure 2). As with the headwind configurations, for a given ground clearance, there is a critical velocity ratio $\left(U^{*}\right)$ below which the ground vortex does not form. However, in the crosswind arrangement the flow field is now characterised by two trailing vortices on the leeward side of the intake.

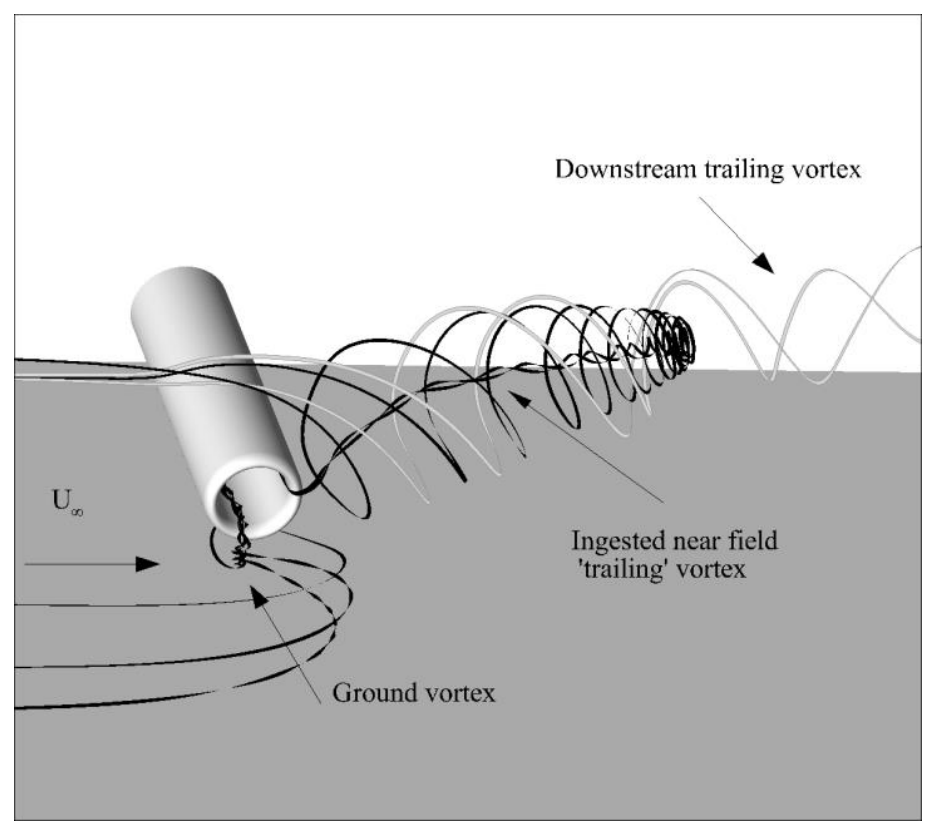

Figure 2: Calculated streamlines under crosswind $\left(h / D_{1}=0.25, U^{*}=19.8, \delta^{*} / D_{1}=0.11\right)$

Although there have been a number of previous studies of ground vortex formation, very few have concentrated on the formation mechanisms and characteristics under tailwind conditions. Bissenger and Braun performed one of the first tailwind studies but the work was limited to small scale water tunnel visualizations [19]. Motycka et al [14, 20, 21] conducted experimental studies with a $1 / 12^{\text {th }}$ scale intake model. Although very little quantitative data was obtained, they suggested that ground vortices which formed under tailwind or reverse thrust conditions were stronger than those formed under quiescent, headwind and forward quarter yaw conditions. Moreover, the trajectories of these vortices were reported to be closer to the intake centre and thus present a greater risk of being ingested into the core of the engine; an event which is known to increase the probability of engine surge.

This study builds on previous experimental [1-5] and computational studies [6, 7] to characterise the ground vortex behaviour under various yawed and tailwind configurations using 
Accepted manuscript

computational fluid dynamics (CFD). The CFD investigations were initially performed for a range of experimentally tested crosswind conditions to establish credibility in the computational approach. Subsequent calculations were performed under tailwind configurations which were beyond the capability of the experimental facility. The results from this study, in conjunction with compatible results from a previous CFD study [6,7], provide a broad assessment of ground vortex characteristics.

\section{Experimental Data}

The experiments conducted by Murphy et al [1-5] were performed in the Cranfield University 2.4 x $1.8 \mathrm{~m}$ low-speed wind tunnel. The model was a generic $1 / 30^{\text {th }}$ scale, axi-symmetric cylindrical intake of uniform cross-section with an internal diameter, $D_{\mathrm{i}}$, of $0.1 \mathrm{~m}$, a highlight diameter, $D_{l}$, of $0.12 \mathrm{~m}$ and an elliptical lip with a major-to-minor axis ratio of 2. The intake did not include a central hub or rotating fan. A suction system provided a massflow of $1.49 \mathrm{~kg} / \mathrm{s}\left(\mathrm{M}_{\mathrm{i}}=0.58\right)$ with a Reynolds number of $1.26 \times 10^{6}$ based on the internal diameter and the average velocity within the intake [1].

Stereoscopic Particle Image Velocimetry (SPIV) was used to measure the three components of velocity on a plane parallel to the ground and beneath the intake at $y / D_{1}=0.083$ from the ground. Total-pressure rakes were used to quantify the flow distortion within the intake. The experiments determined the sensitivity of the ground vortex flow field to yaw angle $(\psi)$, ground clearance $\left(h / D_{1}\right)$, approaching boundary layer thickness $\left(\delta^{*} / D_{1}\right)$ and velocity ratio $\left(U^{*}\right)$. The uncertainties in the experimental measurements included $\pm 1.9-3.6 \%$ uncertainty in setting the non-dimensional height, $\pm 2.64 \%$ uncertainty in velocity ratio for the mean $\mathrm{U}^{*}$ of 6.1 and a worst case uncertainty in non-dimensional vortex circulation of $10 \%$ [4]. Further details of the experimental investigations and a comprehensive uncertainty analysis are presented in Murphy [1]. 


\section{CFD Methodology}

\subsection{Geometry and Grid}

The intake geometry used in the CFD simulations was the same as that in the experimental studies (Figure 3). The overall computational domain, determined from previous CFD studies [6,7], was a rectangular prism with the dimensions $25 D_{1} \times 25 D_{1} \times 12.5 D_{1}$ in the streamwise, spanwise and ground normal directions respectively (Figure 4). The mesh size was approximately 2.1 million hexahedral cells arranged in 58 blocks. The ground plane was set at an $h / D_{\text {, of }} 0.25$. A wall function approach was selected for the near-wall regions and the first cell height was determined to give a $\mathrm{y}^{+}$of approximately 30 .

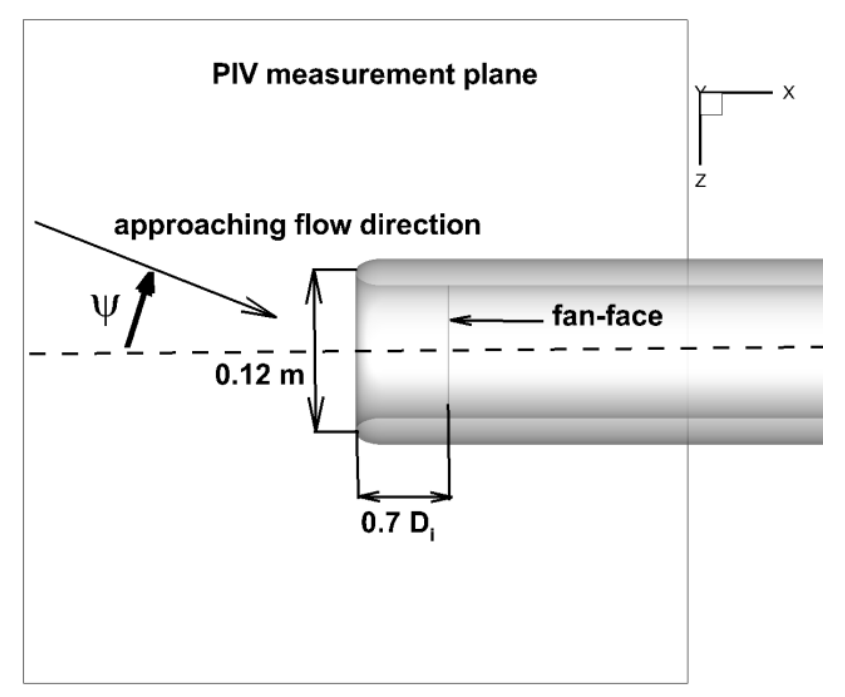

Figure 3: Dimensions of the model intake used in the CFD simulations and definition of yaw angle and the coordinate system 


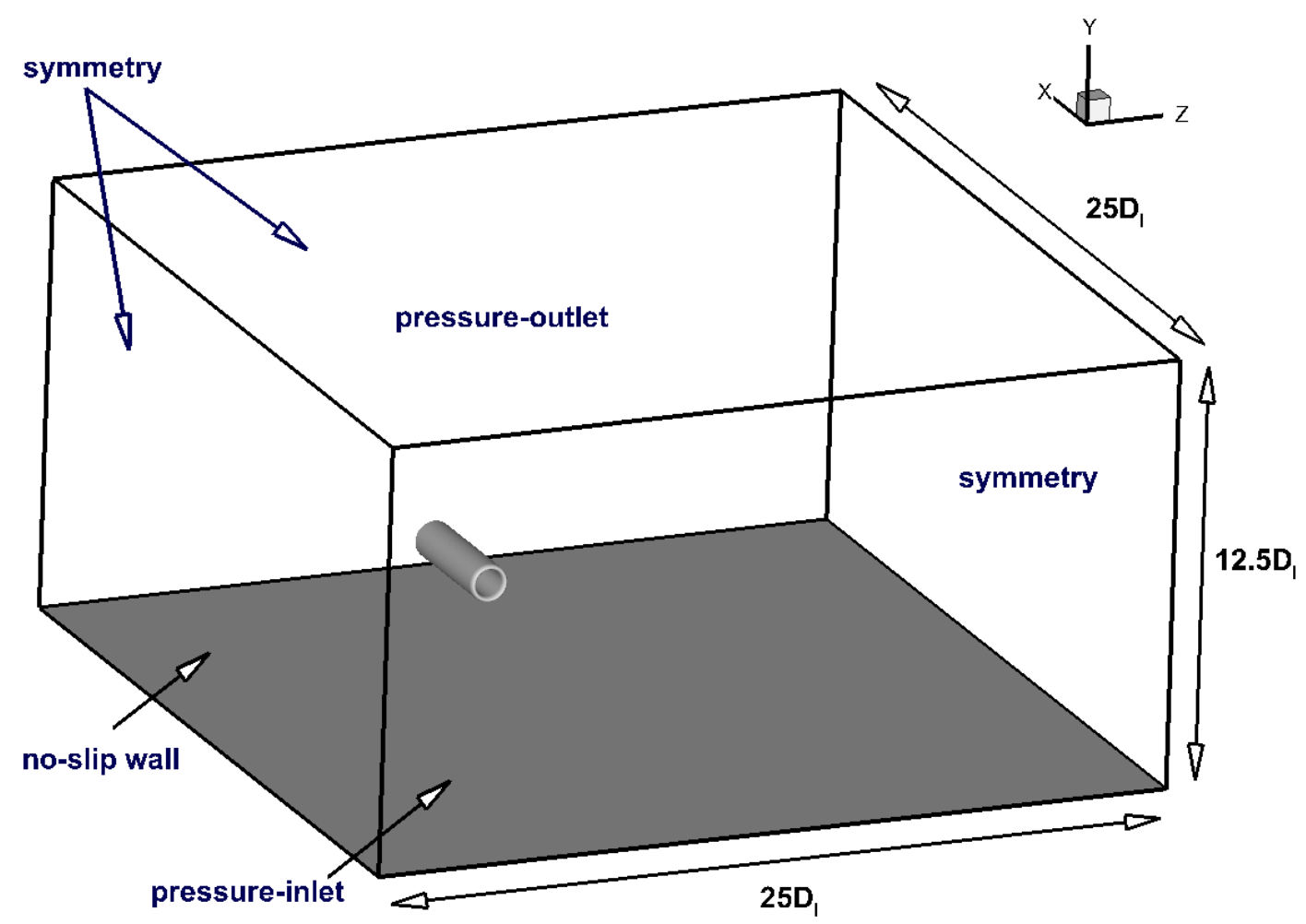

Figure 4: Computational domain and boundary conditions for forward quarter yaw configurations.

\subsection{Boundary Conditions}

. A'pressure inlet' boundary condition was applied to domain inlet (Figure 4). The ground vortex characteristics are known to depend on the vorticity within the approaching boundary layer [2]. Consequently, to enable pertinent comparisons between the experimental and CFD results, the boundary layer total pressure profile at the CFD domain inlet was specified to match the measured boundary layer displacement thickness $\left(\delta^{*} / D_{1}=0.11\right)$ at the intake location.

The total temperature at the inlet was assumed to be a constant and equal to $300 \mathrm{~K}$. This was the static temperature measured in the settling chamber of the wind tunnel which is approximately equivalent to the total temperature. The inlet turbulence was specified in terms of turbulence intensity and a hydraulic diameter of $2 \mathrm{~m}$ which was based on the cross-sectional extents of the wind tunnel. 
The boundary on the downstream side of the domain was specified as a 'pressure outlet' (Figure 4) and the static pressure was set to freestream conditions. The intake suction flow was generated by imposing a static pressure boundary at the exit of the intake duct and a target massflow was specified to match the experiments. The ground plane and intake were defined as no-slip walls in all cases. The 'symmetry' boundary condition was used for the upper surface of the domain. It was also applied to the domain walls parallel to the flow while testing pure headwind, crosswind and tailwind configurations. For the yawed configurations, the two side walls were specified as 'periodic' boundaries (Figure 4).

\subsection{Solver Settings}

The Reynolds Averaged Navier-Stokes (RANS) solver Fluent 12.1 was used to calculate the flow-field. The density based solver was employed with the assumption of an ideal gas. The $3^{\text {rd }}$ Order MUSCL discretisation scheme was implemented along with the k- $\omega$ SST turbulence model. The k- $\omega$ SST model combines the near wall formulation of the standard $k-\omega$ model with the freestream independence of the $k-\varepsilon$ model in the outer part of the boundary layer. This model is reported to be particularly suited for calculating pressure induced separation [22], which is expected to be an important aspect of the ground vortex flow. Jermy and Ho used the k- $\omega$ SST turbulence model for a similar study of intake vortices which arise in jet engine test cells and it was reported to produce similar results to the more computationally expensive Reynolds Stress Models [23]. For the configurations which were simulated using the unsteadyRANS (URANS) approach, an implicit, $2^{\text {nd }}$ order temporal discretisation scheme was employed. A time step of 0.0075 seconds was chosen based on the frequencies observed in Large Eddy Simulations of the ground vortex flow-field conducted by Karlsson \& Fuchs [24]. 500 iterations were performed per time step using a CFL number of 10 and a converged solution was obtained in each case after approximately 70 time steps.

\subsection{Convergence Studies}

As outlined in the introduction, the mechanisms of ground vortex formation, and the associated flow topologies, depend on the freestream yaw angle $(\psi)$ and are different between the headwind and crosswind configurations [1-7].. Therefore, to support the credibility of the 
calculations, grid independence studies were performed for both headwind and crosswind conditions separately following the method of Roache [25]. Three grids comprising approximately $0.9,1.39$ and 2.14 million cells were used in each case. A constant refinement ratio was maintained between the three grids, but the refinement was not uniform between each level. Results from simulations performed on each of these grids showed that vortex strength was not affected appreciably by grid refinement. The change in total vortex circulation was estimated to be $4.7 \%$ between the intermediate and fine grids. The results also showed that, under crosswind conditions, the non-dimensional vortex core radius $\left(r_{c} / D_{i}\right)$ on the PIV measurement plane reduced from 0.22 to 0.19 for the intermediate and fine grids respectively [7]. All simulations presented in this paper have been performed using the finest grid $(2.14$ million cells). Iterative convergence was achieved for all configurations with residuals in the order of $10^{-4}$ or less. In addition, the vortex circulation and intake massflow were also used as convergence indicators where both parameters converged to steady values for all simulations. Further details on the grid convergence studies can be obtained from Zantopp [6-7].

\subsection{Post-Processing}

To directly compare the CFD results with the measurements, the same post-processing techniques were employed for both sets of data. The quantitative results obtained by Murphy [15] were evaluated from velocity data acquired at a plane parallel to the ground (PIV plane at $\left.y / D_{1}=0.083\right)$ and total pressure data within the duct at a location $0.7 D_{i}$ downstream of the highlight. This is equivalent to the 'fan-face' location for a typical civil intake. In keeping with this approach, the CFD results were evaluated from data extracted at these same two key locations and in a similar manner.

The velocity data was used to determine the vortex strength, size and location at the PIV plane. To identify the vortex centre, the $Q$ function was employed (Equation 1), which is a local indicator of the rotation rate compared to the strain rate [26]. The advantage of this method over the vorticity parameter is that it can distinguish between rotational vortex flows, where $Q<0$, and vortical shear layers, where $Q$ becomes 0 . The $Q$-function, along with the maximum (or 
minimum) component of vorticity, was therefore used to identify the vortex centre and sense of rotation respectively.

$$
Q_{x}=\left(\frac{\partial v}{\partial y}-\frac{\partial w}{\partial z}\right)^{2}+\left(\frac{\partial w}{\partial y}+\frac{\partial v}{\partial z}\right)^{2}-\left(\frac{\partial w}{\partial y}-\frac{\partial v}{\partial z}\right)^{2}
$$

As with the experimental results, the CFD data at the PIV plane were interpolated onto a circular grid centred at the vortex centre. The vortex strength, $\Gamma$, was determined by integrating the out of plane vorticity over the entire circular domain and the vortex core size was estimated using the vorticity disc method $[27,1]$.

During the experiments, a set of total pressure rakes were used to characterise the flow field within the intake. For each configuration, measurements were taken at 72 and 9 equi-spaced circumferential and radial locations respectively. The CFD data were interpolated onto the same grid size at the fan face to enable direct comparisons. To quantify the distortion resulting from vortex ingestion, the $D C(60)$ coefficient was employed (Equation 2).DC(60) is defined as the difference between the average total pressure at the fan-face and the minimum average total pressure in a $60^{\circ}$ sector, non-dimensionalised by the in-duct dynamic head.

$$
D C(60)=\frac{P_{0 f}-P_{060}}{q_{f}}
$$

The impact of ground vortex ingestion on the swirl velocity profile has also been quantified in terms of $\mathrm{SC}(60)$ (Equation 3). $\mathrm{SC}(60)$ is defined as the difference between the maximum average swirl velocity in a $60^{\circ}$ sector and the average swirl velocity on the fan-face, nondimensionalised by the in-duct axial velocity.

$$
S C(60)=\frac{\left|U_{\theta, 60}\right|-\left|U_{\theta, f}\right|}{U_{a x}}
$$

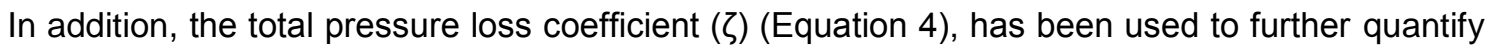
the in-duct flowfield. $\zeta$ is defined as the difference between the freestream total pressure and the average total pressure at the fan-face, non-dimensionalised by the in-duct dynamic head.

$$
\zeta=\frac{P_{0 \infty}-P_{0 f}}{q_{f}}
$$




\section{Accepted manuscript}

\section{CFD Validation}

Experiments conducted by Murphy et al investigated the ground vortex flow field at various yaw angles ranging from $0^{\circ}$ to $90^{\circ}$ at a single velocity ratio $\left(U^{*}=19.8\right)$, non-dimensional height $\left(h / D_{1}=0.25\right)$ and approaching boundary layer thickness $\left(\delta^{*} / D_{1}=0.11\right)[1-5]$. Figure 5 compares the measured and computed vorticity contours and streamline patterns for increasing yaw angle. The experiments show that as the approaching wind yaw angle $(\psi)$ increases from 30 to 90 degrees the peak vorticity magnitude increases by a factor of approximately 2.4 (Figure 5b,c). This is a result of the vorticity source changing from being predominantly associated with the approaching boundary layer to being related to the boundary layer over the external intake surface. As the yaw angle $(\psi)$ increases, in addition to the increase in strength, there is an associated increase in the size of the ingested ground vortex and also a change in its position (Figure 5). The CFD calculations, shown in Figures 5(d)-(f), agree with these experimental observations of the flow features both in terms of vorticity footprint and the vortex location relative to the intake. 


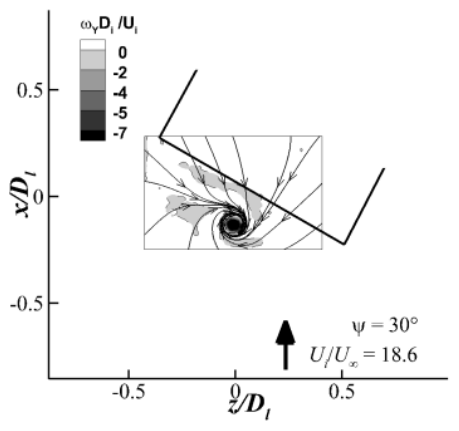

(a)

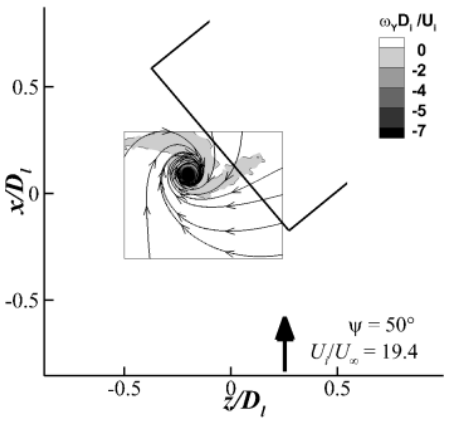

(b)

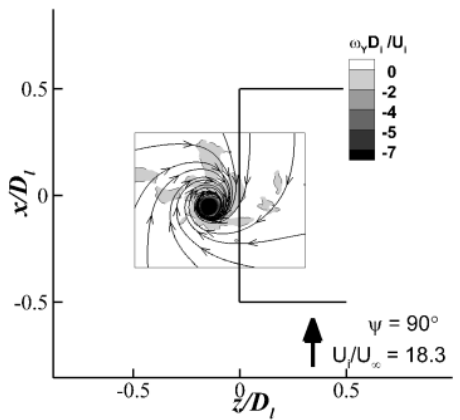

(c)

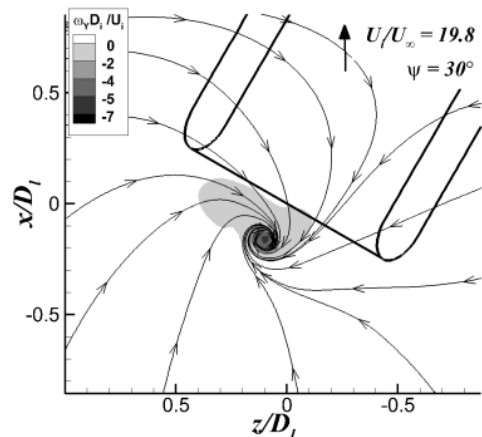

(d)

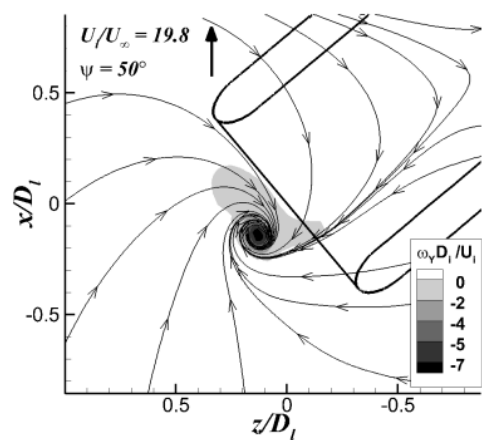

(e)

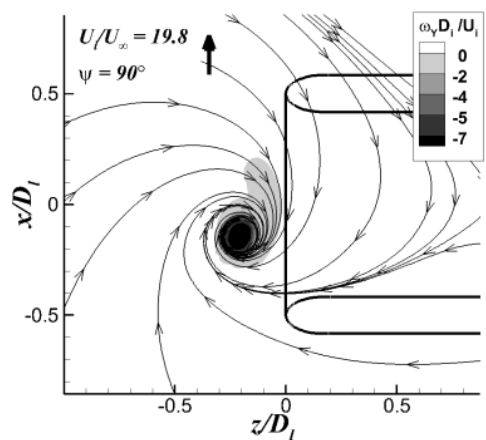

(f)

Figure 5: The effect of yaw angle on the ground vortex flow-field. Contours of component vorticity superimposed by streamlines on the PIV plane (a)-(c) measurements (d)-(e) CFD ( $h / D_{1}=0.25$, $\left.U^{*}=19.8, \delta^{*} / D_{1}=0.11\right)$

Figure 6 compares the calculated and measured variation of the average non-dimensional vortex strength $\left(\Gamma^{*}\right)$ with yaw angle. In general, the CFD calculations capture the correct trend in which there is a monotonic increase in the total non-dimensional vortex circulation as the yaw angle is increased. For the pure crosswind case $\left(\psi=90^{\circ}\right)$, the difference is $0.8 \%$. Overall the 


\section{Accepted manuscript}

calculated values of vortex strength $\left(\Gamma^{*}\right)$ for the yaw configurations are in reasonable agreement with the experimental dataset with a maximum difference of $13.5 \%$ in total circulation (Figure 6). The relatively large discrepancies at 0 and $70^{\circ}$ could be due to a number of factors. At a yaw angle of $0^{\circ}$ the difference could be due to the model's inability to accurately calculate the suction induced vorticity source which is the dominant aspect at this yaw angle and velocity ratio. Furthermore, for this configuration $\left(\psi=0^{\circ}, h / D_{1}=0.25, U^{*} \sim 20\right.$ and $\left.\delta^{*} / D_{1}=0.11\right)$, Murphy observed that the dominant vorticity source switches from between being associated with the induced and the approaching flowfields [1]. The complicated and unsteady flow physics at this transition velocity ratio therefore may not be fully captured in the CFD calculations.

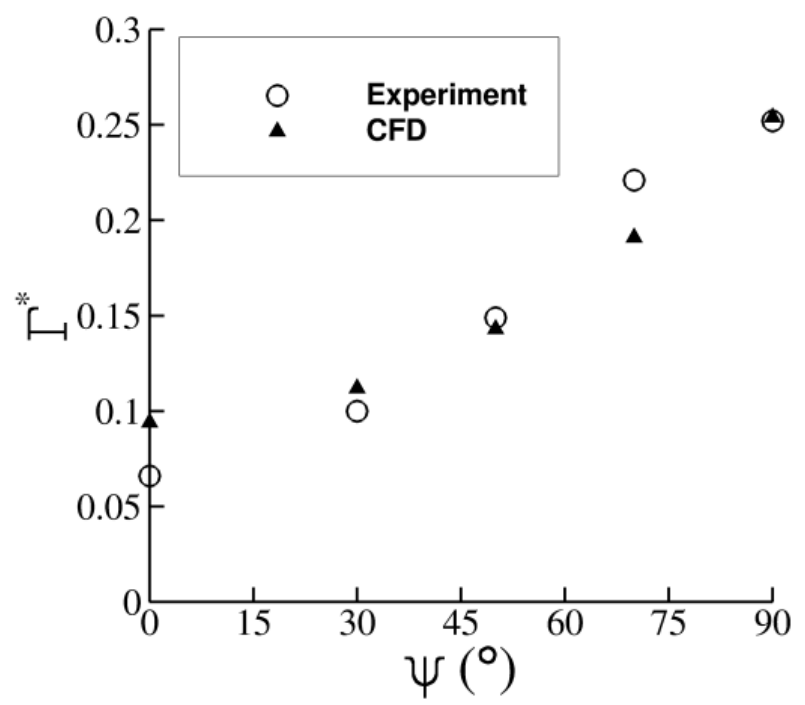

Figure 6: Variation of PIV plane non-dimensional circulation with yaw angle - CFD vs. Experiment $\left(h / D_{1}=0.25, U^{*}=19.8, \delta * / D_{1}=0.11\right)$

The yaw angle also has a notable impact on the fan-face total pressure distribution and flow distortion. The total pressure measurements show that as the yaw angle increases, the size of the vortex footprint and the peak loss increase at the fan face [1-5]. The intake distortion is directly proportional to both these changes and as a result, the quality of the flow entering the intake deteriorates with yaw angle when a ground vortex is present. Figure 7 compares the total pressure distribution at a velocity ratio of 19.8 and yaw angle of $70^{\circ}$. The CFD calculations of the flow-field show reasonable topological agreement with the experiments in terms of the location and size of the vortex. 


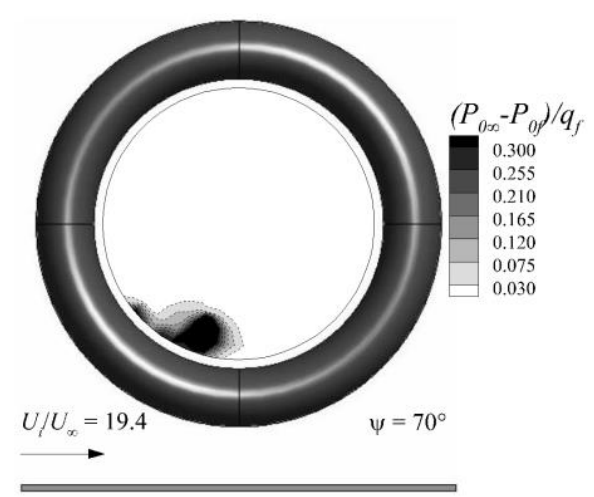

(a)

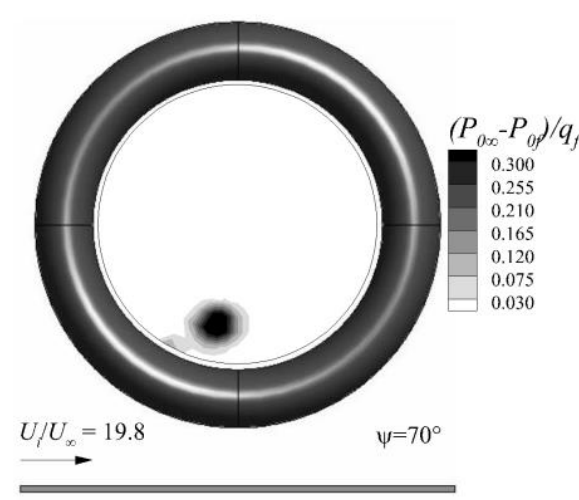

(b)

Figure 7: Fan-face total pressure distribution (a) experiment (b) $C F D\left(\Psi=70^{\circ}, h / D_{1}=0.25, U^{*}=19.8\right.$, $\left.\delta^{*} / D_{1}=0.11\right)$

The above findings demonstrate that the CFD method implemented in the present study captures the main sensitivities of the ground vortex flowfield for a range of yaw angle settings. In addition, a previous study by Zantopp [6], which employed the same CFD method, successfully captured the flow field under quiescent, headwind and crosswind conditions for a variety of velocity ratio and ground clearance configurations.

\section{Tailwind and Rear Quarter Yaw Flow Topologies}

The aim of this part of the investigation is to apply the computational method to configurations that were beyond the scope of the experimental test facility. Simulations have been carried out at various velocity ratios under pure tailwind $\left(\Psi=180^{\circ}\right)$ and rear quarter $\left(\Psi=150^{\circ}\right)$ conditions and an analysis of these calculations has been conducted to identify the main characteristics of the flow-field as well as its variation with streamtube velocity ratio $\left(U^{*}\right)$.

\subsection{Tailwind $\left(\Psi=180^{\circ}\right)$}

Figure 8 compares the flow topology between the headwind $\left(\Psi=0^{\circ}, U^{*}=9.9\right)$ and tailwind $\left(\Psi=180^{\circ}, U^{*}=9.9\right)$ configurations at a fixed non-dimensional height. For the pure tailwind case (Figure 8b), the CFD method calculates a steady, symmetrical flow field with a pair of counter rotating ground vortices. The solution passes through an initial start-up transient, but locks into a steady state after $30-40$ time steps. This is different from the CFD calculations under 
headwind conditions where the flow field remains unsteady. The same general vortex topology and steadiness was observed for most velocity ratios. As expected, the sense of rotation of the vortices under this tailwind arrangement is the same as that under quiescent conditions and opposite to that under headwind conditions (Figure 8). This is due to the approaching vorticity, associated with the tailwind flow, emanating from the same direction as the dominant vorticity source under no-wind conditions (Figure 1a).

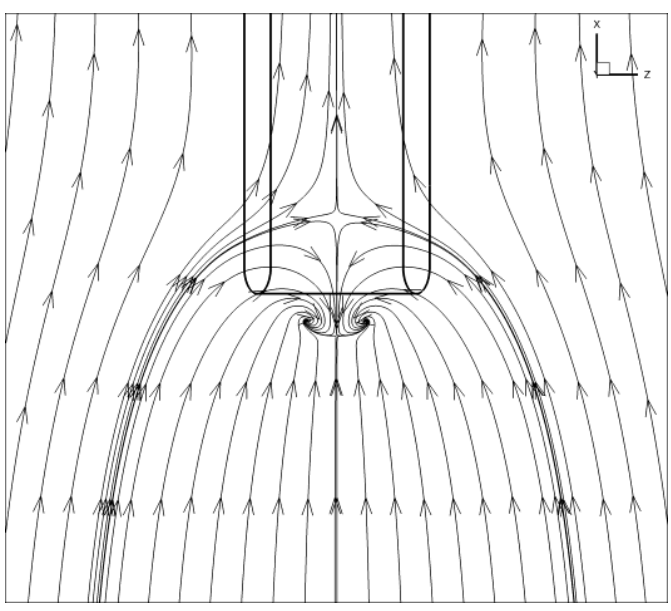

(a) $\mathrm{U}^{*}=9.9 ; \psi=0^{\circ}$

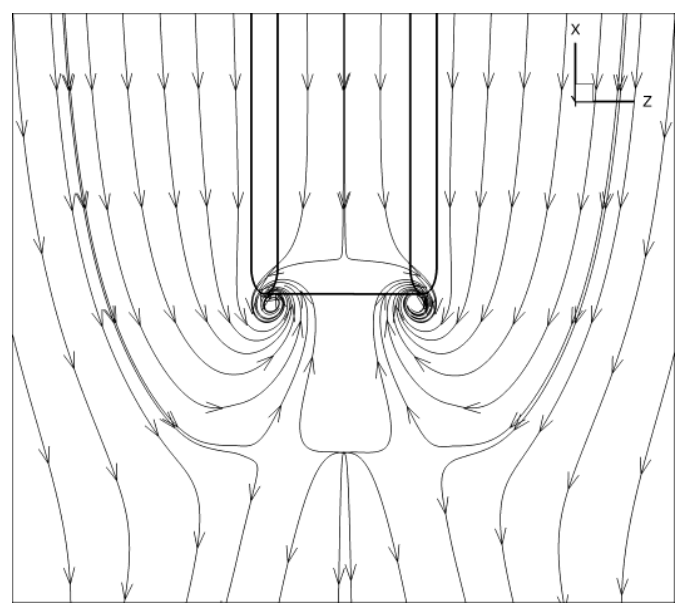

(b) $U^{*}=9.9 ; \psi=180^{\circ}$

Figure 8: Comparison between the time-averaged/steady calculated ground vortex flow-fields under (a) headwind and (b) tailwind conditions $\left(h / D_{1}=0.25\right)$ at the PIV plane

A notable observation is that the distance separating the centres of the ingested vortices at the PIV plane is substantially larger for the tailwind condition in comparison with the headwind arrangement (Figure 8). This has an effect on the vortex ingestion location within the duct which is presented in Section 5.2. Figure 8 also shows that the approaching sucked streamtube size for the tailwind configuration is approximately $0.5 D_{l}$ wider in comparison to the headwind case. This is due to the presence of the intake which introduces a blockage which forces the intake to draw additional mass-flow from over a broader area. Thus for a given velocity ratio the tailwind configuration is likely to ingest a greater level of approaching boundary layer vorticity which will contribute to a stronger vortex. 


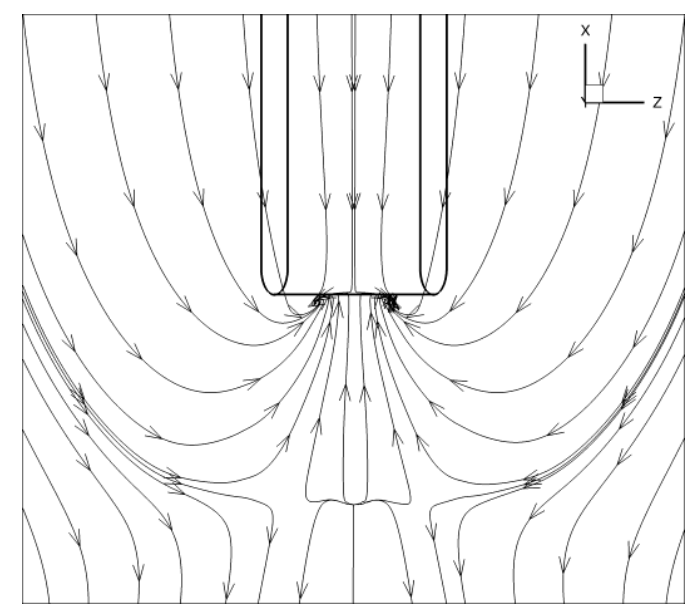

(a) $U^{*}=19.8$

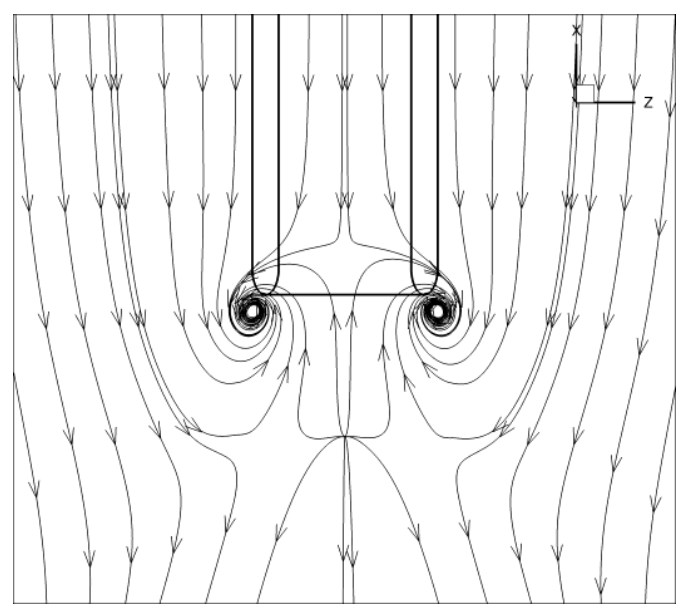

(b) $\mathrm{U}^{*}=6.6$

Figure 9: Calculated streamlines on the PIV reference plane under tailwind conditions $\left(\Psi=180^{\circ}\right)$ for (a) $U^{*}=19.8$ (b) $6.6\left(h / D_{1}=0.25, \delta^{*} / D_{1}=0.11\right)$

Figure 9 compares the vortex topology for a velocity ratio of 19.8 and 6.6 and shows that as the velocity ratio reduces the vortices move away from each other. As $U_{\infty}$ increases, and $U^{*}$ reduces, the size of the sucked streamtube decreases and the node and saddle points, which form ahead of the intake, are drawn closer to the highlight plane. This results in a greater separation between the vortex focal points and a change in the location of the vortex ingestion location. At $U^{*}=19.8$, the suction induced boundary layer vorticity from ahead of the intake and the approaching boundary layer vorticity oppose each other and the resulting ground vortices are relatively weak and small in size. At a velocity ratio of $U^{*}=6.6$ the increased level of freestream vorticity dominates the flow-field and results in a clearly formed strong ground vortex system.

\subsection{Rear Quarter Yaw $\left(\Psi=150^{\circ}\right)$}

Ground vortex formation under $150^{\circ}$ yaw conditions has been studied at various velocity ratios $\left(U^{*}=19.8,9.9,6.6,4.95,3.96\right)$. As with the pure tailwind configuration, the ground vortex flow field is found to be steady at all velocity ratios. Similar to the forward quarter yaw arrangement (Section 4), the flow-field comprises a single ground vortex (Figure 10). However this vortex rotates in the opposite sense and is accompanied by one or more trailing vortices (Figure 10), in which the strength, size, rotation and orientation all vary with velocity ratio. The presence of the trailing vortex for this configuration was anticipated given the formation mechanism of a similar 


\section{Accepted manuscript}

vortex under pure cross wind $\left(\Psi=90^{\circ}\right)$ conditions where the flow over the intake, and within the sucked streamtube, produces vorticity which is responsible for the formation of the trailing vortex. Since the interaction of the approaching flow with the intake is much greater for a yaw angle of $150^{\circ}$ in comparison to the forward quarter cases it is credible that a trailing vortex forms for the former but not for the latter.

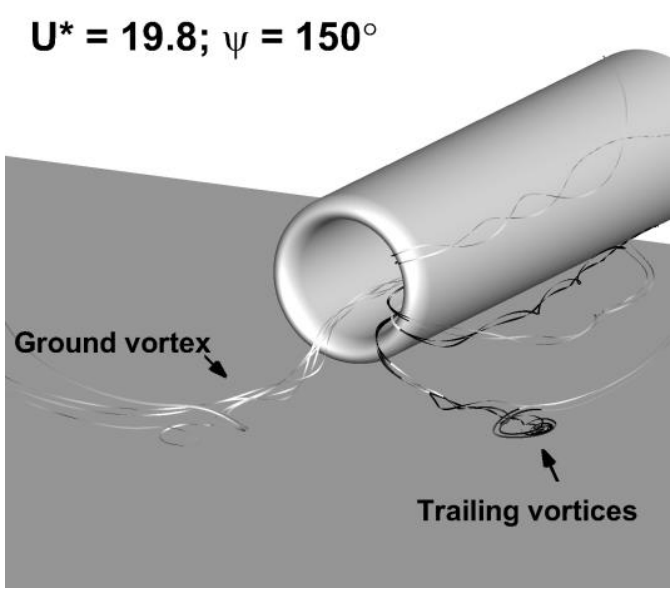

(a)

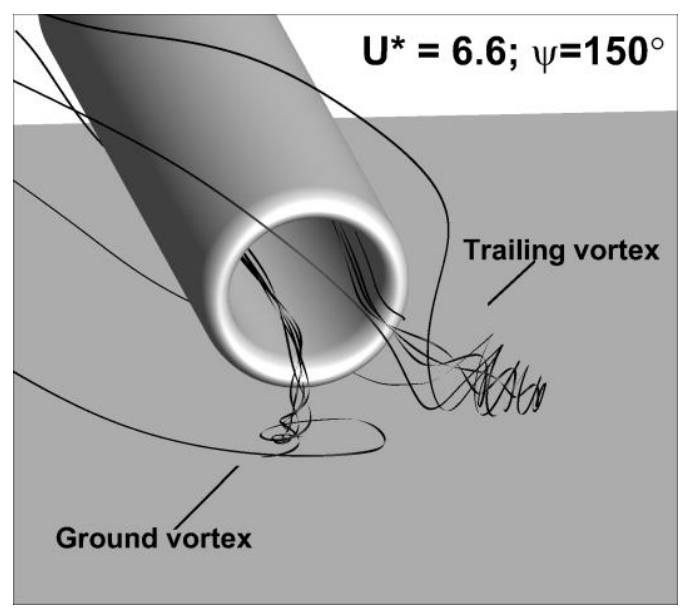

(b)

Figure 10: Ground vortex flow-field visualisation under $150^{\circ}$ yaw conditions for $(a) U^{*}=19.8$ (b) $U^{*}=$ $6.6\left(h / D_{1}=0.25, \delta^{*} / D_{1}=0.11\right)$

At a velocity ratio of 19.8 , a positive $\omega_{x}$ ground vortex is ingested at approximately the 7 o'clock fan face position (Figure 10a and Figure 11) which is accompanied by a relatively weaker and smaller trailing vortex. This trailing vortex is ingested closer to the intake surface at the 4 o'clock position along with a system of smaller trailing vortices (Figure 10a and Figure 11). This flowfield is in agreement with the experimental observations of Bissinger \& Braun [19]. When the velocity ratio is reduced to $U^{*}=6.6$ by increasing the freestream velocity, the smaller trailing vortices are no longer observed as the sucked streamtube reduces its interaction with the intake outer surface. The flow-field within the duct now comprises a positive $\omega_{x}$ ground vortex and a negative $\omega_{x}$ trailing vortex (Figure 10b). In comparison to the $U^{*}=19.8$ configuration, both vortices are stronger with the ground vortex still notably stronger than the trailing vortex.

With a further reduction in the velocity ratio to 3.96 , a switch in the sense of rotation of the two vortices is observed. The ground vortex now has negative $\omega_{x}$ vorticity while the trailing vortex 


\section{Accepted manuscript}

has positive $\omega_{\mathrm{x}}$ vorticity. Murphy observed similar characteristics experimentally under pure crosswind when the velocity ratio was close to the vortex blow-away point [1]. At this velocity ratio $\left(U^{*}=3.96\right)$, the interaction between the capture streamtube of the intake and the ground plane is greatly reduced. As a result, the influence of the vorticity contained within the approaching boundary layer on the ground vortex formation is also reduced. The vorticity from the suction induced boundary layer from ahead of the intake therefore becomes dominant and this results in a change in rotation of the ground vortex. At this velocity ratio $\left(U^{*}=3.96\right)$ the trailing vortex is calculated to be the stronger of the two vortices.

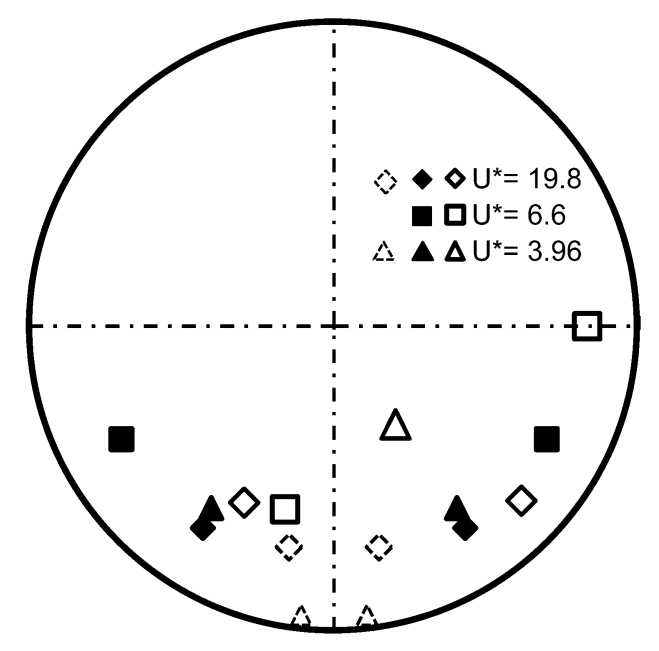

Figure 11: Impact of velocity ratio on the ingestion location of vortex centres at the fan face under tailwind ( $\Psi=180^{\circ}$, solid symbols),yawed conditions ( $\Psi=150^{\circ}$, open symbols) and headwind conditions $\left(\Psi=0^{\circ}\right.$, dashed symbols $)\left(h / D_{1}=0.25, \delta^{*} / D_{1}=0.11\right)$.

The location of the ingested vortices depends on both the yaw angle as well as the velocity ratio (Figure 11). For a bypass engine one of the key concerns is vortex ingestion into the engine core and therefore the vortex radial location is of particular interest. For the tailwind cases, although the circumferential position changes with velocity ratio, the radial position is relatively insensitive. For the $\psi=150^{\circ}$ configurations, the radial positions are also depend on the velocity ratio but the main observation is that for the case closest to the blow away condition $\left(U^{*}=3.96\right)$ the vortex ingestion occurs much closer to the engine centreline. 


\section{Ground Vortex Characteristics}

The results from the present work, together with data from a compatible study [6,7], enable a broad assessment of the ground vortex systems. The vortex characteristics, in terms of the vortex strength and the effect on the intake flow distortion, are considered for a range of headwind, front quarter, read quarter and tailwind arrangements. All data used to develop these trends comes from simulations carried out at a non-dimensional height $\left(h / D_{1}\right)$ and boundary layer thickness $\left(\delta^{*} D_{1}\right)$ at the intake position of 0.25 and 0.11 , respectively.

\subsection{Vortex Strength}

From the flow field investigations it is clear that the ground vortex flow modes strongly depend on the yaw angle and velocity ratio. Indeed, the intake flow structure comprises single or multiple vortices and the size, strength and rotation sense of these ground vortices depends on the configuration. The effect of velocity ratio and yaw angle on the calculated non-dimensional circulation at the PIV measurement plane is shown in Error! Reference source not found.. Overall, the results show that as the velocity ratio decreases, the total circulation increases until a local maximum is reached. A further reduction in velocity ratio leads to a reduction in the vortex strength until the critical velocity ratio $\left(U_{\text {crit }}\right)$ is reached. At this point the sucked streamtube no longer interacts with the ground plane and the vortices are 'blown-away'. This agrees with the experimental observations of Murphy under pure headwind and crosswind conditions [1]. The variation of vortex strength with velocity ratio arises from two competing mechanisms. As the freestream velocity increases the vorticity within the approaching boundary layer increases. However, simultaneously the size of the sucked streamtube reduces and therefore the extent of the ingested boundary layer also reduces [2]. The local maximum in terms of the velocity ratio depends on the specific configuration (Error! Reference source not found.). The results tend to group into two modes: relatively weaker ground vortices which form under headwind and $\psi=150^{\circ}$ conditions and stronger vortices which occur under front quarter $\left(\psi=70^{\circ}\right)$, pure crosswind $\left(\psi=90^{\circ}\right)$ and tailwind $\left(\psi=180^{\circ}\right)$ conditions. The differences in total nondimensional circulation between these two groups are more pronounced at lower velocity ratios $\left(U^{*}<10\right)$. 


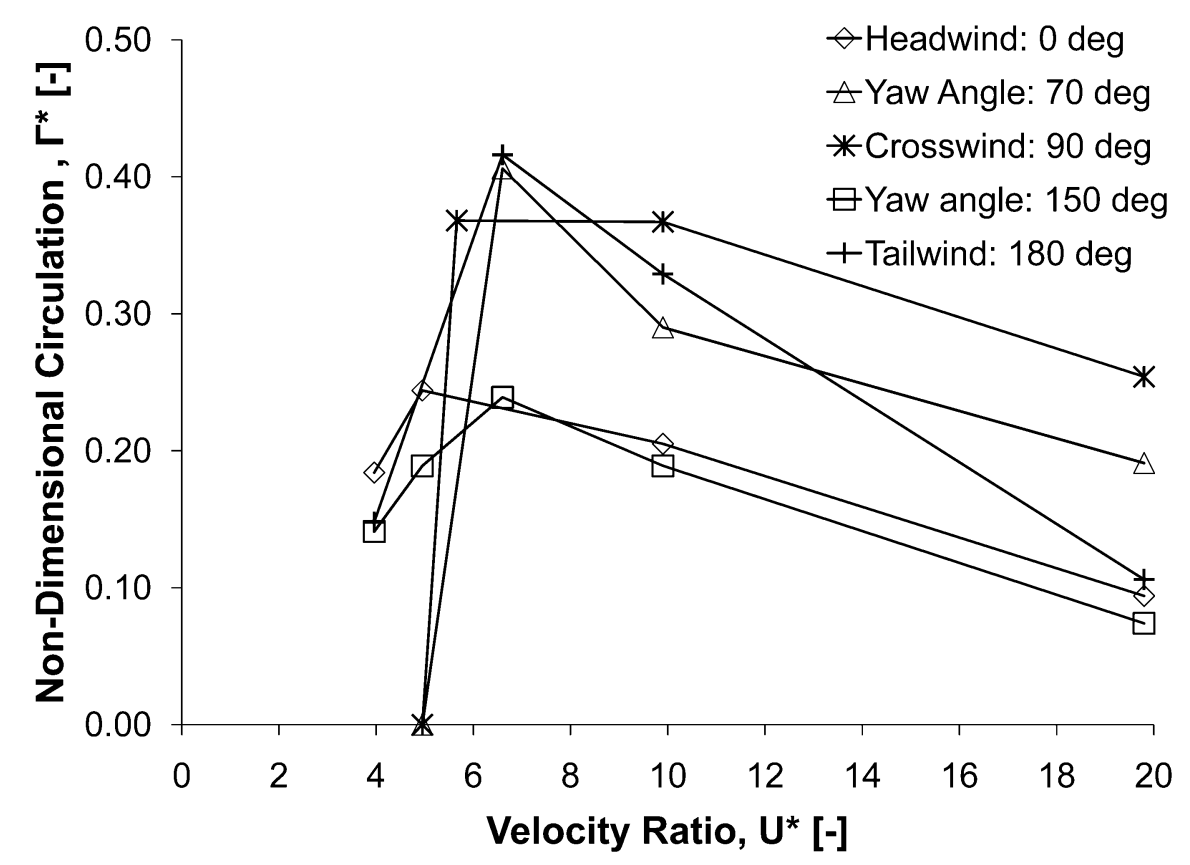

Figure 12: Influence of velocity ratio and yaw angle on non-dimensional circulation at the PIV measurement plane $\left(h / D_{l}=0.25, \delta * / D_{1}=0.11\right)$

Of particular interest is the difference between the tailwind and headwind characteristics. For a large $U^{*}$, there is very little difference between the vortex strength of these cases. Furthermore, they have similar vortex strength at lower $U^{*}$ where the blow-away condition is being approached. However, in the intermediate velocity ratio range, the tailwind configuration results in a ground vortex with almost twice the strength of the headwind cases. For these configurations, the vortex strength mostly depends on the suction induced vorticity and the vorticity of the approaching flow. At a large velocity ratio, the flow field is dominated by the suction induced vorticity. Therefore, as the velocity ratio is increased the difference in the vortex strength between the headwind and tailwind configurations reduces as the flow field tends towards that which arises under quiescent conditions. As the freestream velocity increases, the vorticity within the approaching boundary layer grows causing a corresponding rise in the strength of the ground vortices ingested until a local maximum in circulation is reached. Under a headwind, the suction induced and approaching freestream boundary layers arise in opposite directions and so the vorticity from one source acts in opposition to that from the other. Under tailwind conditions however, both these sources of vorticity approach from the same direction (i.e. from behind the intake highlight) and therefore combine together. Reducing the tailwind velocity ratio $\left(U^{\star}\right)$ to intermediate levels therefore has a greater impact when compared to 
headwind conditions. Although the strongest vortex occurs under tailwind conditions (Error! Reference source not found.), it is unlikely this local maximum would be encountered in a fullscale engine where, due to the take-off operating restrictions under tailwind conditions, the velocity ratio is unlikely to be less than 10 .

\subsection{Intake Distortion Coefficients}

In the current work, the total pressure distortion coefficient $\mathrm{DC}(60)$, the swirl velocity distortion coefficient $\mathrm{SC}(60)$ and the total pressure loss coefficient $(\zeta)$ are analysed at the nominal fan face location.

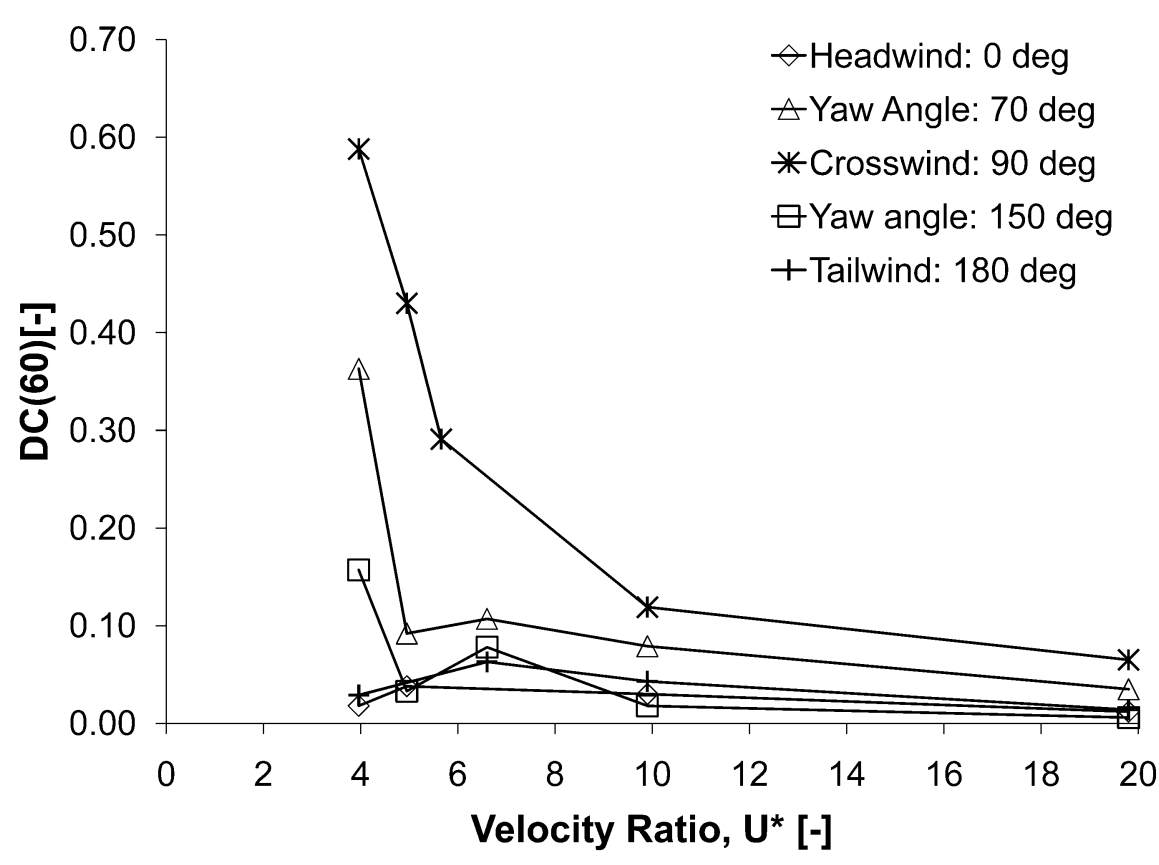

Figure 13: Influence of velocity ratio and yaw angle on $D C(60)\left(h / D_{1}=0.25, \delta^{*} / D_{l}=0.11\right)$

Figure 13 shows the $\mathrm{DC}(60)$ coefficient against velocity ratio for the range of yaw angles investigated from 0 to 180 degrees. The level of fan-face distortion is highest under crosswind conditions for all velocity ratios where a monotonic rise in $\mathrm{DC}(60)$ is observed as $U^{*}$ reduces. For the crosswind configuration, the initial increase in $\mathrm{DC}(60)$ is primarily associated with the ingested crossflow vortex. However, at low velocity ratios $\left(U^{*}<10\right)$, the flow distortion is dominated by a large separation on the windward side of the intake lip which results in the rapid 
increase in $\mathrm{DC}(60)$. This is in contrast with the vortex strength characteristic where $\Gamma^{*}$ reduces below a velocity ratio of 5.65 (Error! Reference source not found.). The front quarter yawed configuration $\left(\psi=70^{\circ}\right)$, also shows relatively high values of $\mathrm{DC}(60)$, although the trend is nonmonotonic with reducing velocity ratio $\left(\mathrm{U}^{\star}\right)$. In this case, the $\mathrm{DC}(60)$ shows a local maximum at the point where the ground vortex is the strongest $\left(U^{*}=6.6\right)$ (Error! Reference source not found.), before similarly exhibiting a large increase in $\mathrm{DC}(60)$ as the intake lip flow separates below a $U^{*}<$ of 4.95 .-For higher velocity ratios (e.g. $U^{*}=9.9$ and 19.8 ), $\psi=90^{\circ}$ and $\psi=70^{\circ}$ still record the highest values although there is no longer any significant crosswind lip separation.

The next most potent configuration, after the pure crosswind and the front quarter yaw $\left(\psi=70^{\circ}\right)$ arrangements, is the rear-quarter case $\left(\psi=150^{\circ}\right)$ where the local maximum occurs at the velocity ratio where the ground vortex is strongest $\left(U^{*}=6.6\right)$. As the velocity ratio is further reduced and the lip loading is increased, the flow separates on the windward side of the intake and there is a large increase in the $\mathrm{DC}(60)$ (Figure 13). In contrast to the vortex strength characteristics (Error! Reference source not found.), the tailwind configuration is more benign based on the $\mathrm{DC}(60)$ metric. This is a result of the overall reduction in average total pressure at fan-face caused by the ingested vortex pair. This reduction masks the total-pressure loss recorded in the 'worst' $60^{\circ}$ sector and highlights the limitation of the $\mathrm{DC}(60)$ distortion descriptor. For the tailwind case, as there is no gross lip separation, the variation in $\mathrm{DC}(60)$ with $U^{*}$ follows the same trend as $\Gamma^{*}$ with a local maximum at $U^{*}=6.6$.

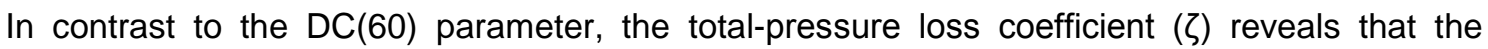
tailwind configuration $\left(\psi=180^{\circ}\right)$ has the greatest loss across all the velocity ratios where a monotonic increase is observed as the velocity ratio reduces (Figure 14). Similarly, for the crosswind configuration $\left(\psi=90^{\circ}\right)$, the loss increases with reducing velocity ratio and reaches a similar level to the tailwind configuration at the lowest velocity ratio of 3.96 . However, the flow fields associated with the two configurations are different where for the tailwind case the loss is dominated by the contra-rotating ingested vortices, for the crosswind configuration the loss is due to both the windward lip flow separation as well as the ingested single ground vortex [6-7]. The magnitude of the loss reduces as the yaw angle decreases although there is still a notable 


\section{Accepted manuscript}

level of loss for $\psi=70^{\circ}$. However, the rear quarter configuration of $\psi=150^{\circ}$ is characteristically different from the other yawed cases. As the velocity ratio $\left(U^{\star}\right)$ is reduced from 9.9 to 6.6 , there is a rapid rise in the loss which is mostly due to the increase in the size of the ingested ground vortex. For a lower velocity ratio of 4.95 there is a reduction in $\zeta$ which is a result of the smaller size of the ingested streamtube leading to a reduction in the interaction with the ground plane and consequently a reduction in the size of the ground vortex. Finally, as the velocity ratio is further reduced $\left(U^{*}=3.96\right)$, the loss increases again and is due to the increase in the local flow turning around the intake lip as it is ingested and results in an internal flow separation on the windward side of the duct.

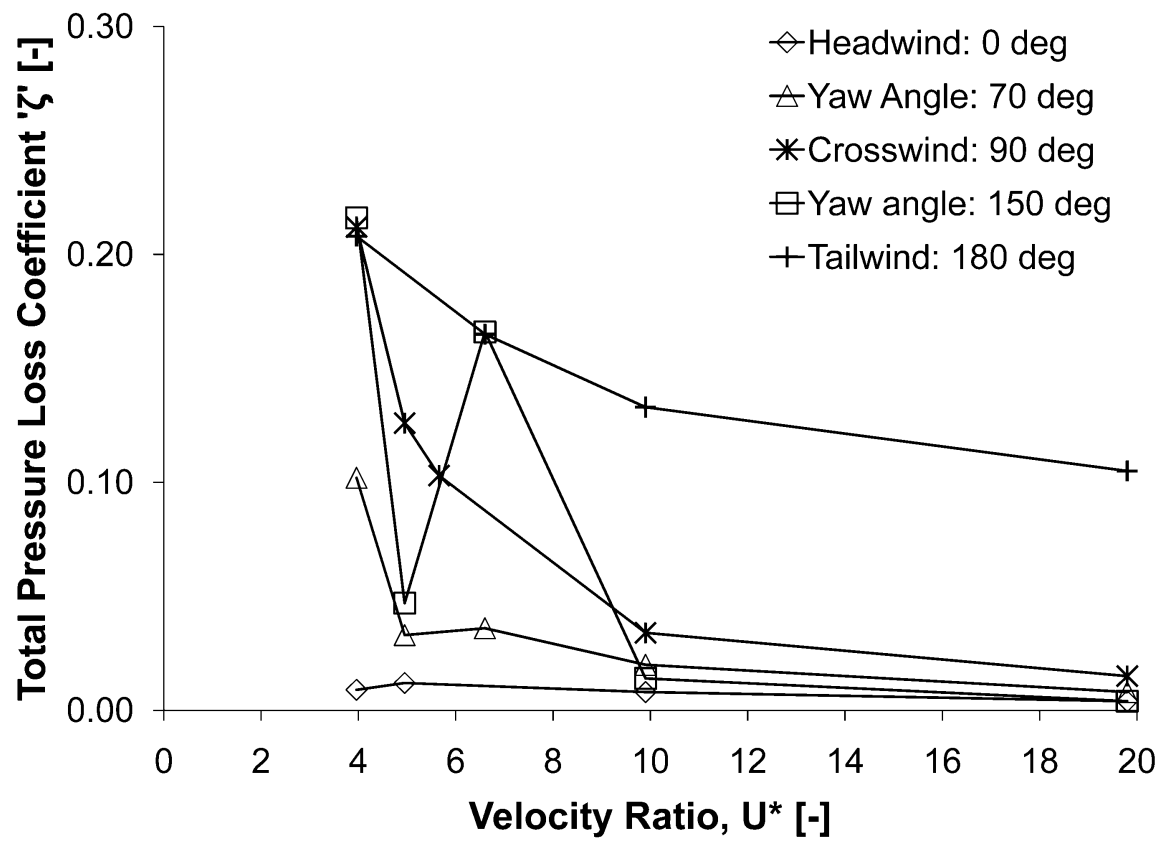

Figure 14: Influence of velocity ratio and yaw angle on total-pressure loss coefficient $\left(h / D_{1}=0.25\right.$, $\left.\delta * / D_{1}=0.11\right)$

Although $\mathrm{DC}(60)$ is a relatively common parameter which is used to quantify the level of intake distortion, it has several limitations. $\mathrm{DC}(60)$ is based on total-pressure distributions and the limitations of this approach are particularly acute for flow distortions characterised by flow angle non-uniformities. This is the nature of the flow fields under consideration here where the intake aerodynamics is dominated by compact vortices. For example in a tailwind configuration, the ingested ground vortex produces regions where the local swirl angle;

$$
\beta=\frac{V_{a x}}{V_{\theta}}
$$


in the intake varies by $\pm 21^{\circ}$ across the relatively small region of the vortex. This distortion of the velocity field angularity is considered in terms of $\mathrm{SC}(60)$ (Equation 3). As expected, the greatest $\mathrm{SC}(60)$ is encountered at low velocity ratios for the crosswind configuration where lip separation dominates the flow. In this case the separation results in a large deficit in the axial velocity $\left(\mathrm{V}_{\mathrm{ax}}\right)$ and therefore even a modest circumferential velocity results in a large swirl angle ( $\beta)$. SC(60) does highlight some key differences in comparison to the $\mathrm{DC}(60)$ descriptor. The variation in distortion levels at higher velocity ratios are more notable in terms of $\mathrm{SC}(60)$ (Figure 15) when compared with $\mathrm{DC}(60)$ (Figure 13). As is the case with total-pressure distortion (Figure 13), the peak values for swirl distortion are also encountered for the crosswind case where it is dominated by the windward lip separation. For the tailwind case $\left(\psi=180^{\circ}\right)$, the SC(60) steadily increases as the velocity ratio is reduced although the levels are typically less than half of those calculated for the $\psi=90^{\circ}$ case (Figure 15). The rear quarter configuration $(\psi$ $\left.=150^{\circ}\right)$ typically has higher $\mathrm{SC}(60)$ levels than the tailwind configuration although still notably lower than the crosswind case. The only exception to these observations is at low velocity ratios $\left(\mathrm{U}^{*}<5.5\right)$ where there is a large reduction in $\mathrm{SC}(60)$. This is where the crosswind ground vortex has almost reached the blow-away condition but the $\mathrm{DC}(60)$ is still relatively large due to lip separation (Figure 13).

There is a general decrease in $\mathrm{SC}(60)$ for the forward quarter cases as the yaw angle is reduced from $\psi=90^{\circ}$ to the pure headwind case $\left(\psi=0^{\circ}\right)$ and not surprisingly the $\operatorname{SC}(60)$ has similar characteristics to the $\Gamma^{*}$ distributions. When considering the key condition of a large velocity ratio $\left(U^{*} \sim 20\right)$, the $S C(60)$ results are grouped in three regions. The singular worst configuration is the crosswind case and the most benign are the pure headwind and pure tailwind cases - however, even in these cases the calculated local swirl angle is up to $10^{\circ}$ within the ingested vortex region. 


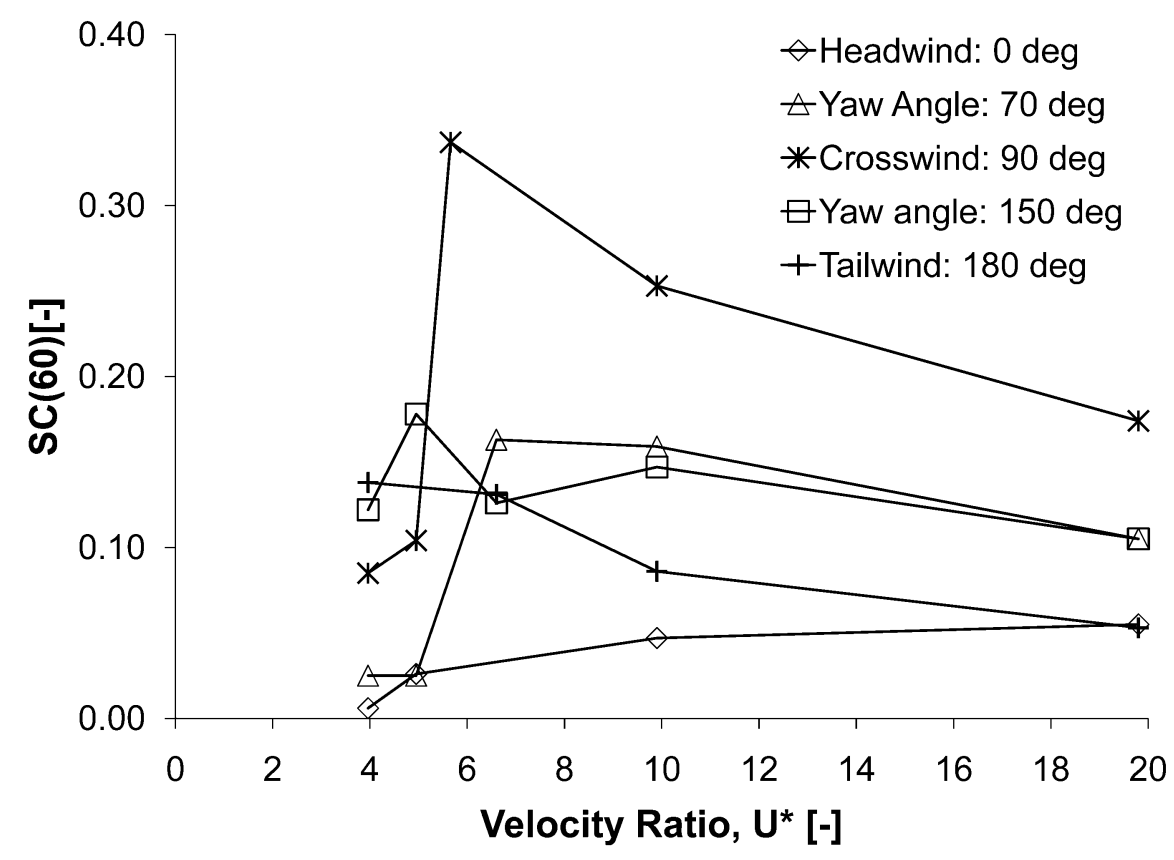

Figure 15: Influence of velocity ratio and yaw angle on $\operatorname{SC}(60)\left(h / D_{1}=0.25, \delta^{*} / D_{1}=0.11\right)$

\section{Summary and Conclusions}

The characteristics of intake ground vortices has been investigated for a range of approaching wind angles $(\Psi)$ and intake velocity ratios $\left(\mathrm{U}^{*}\right)$ using a RANS based computational method. The calculations were validated against an experimental dataset for forward quarter yaw configurations $\left(0^{\circ}<\psi<90^{\circ}\right)$ in which the basic trends were captured. Focus was then concentrated on establishing the ground vortex characteristics for rear quarter yaw $\left(\psi=150^{\circ}\right)$ and tailwind cases $\left(\psi=180^{\circ}\right)$ - configurations that represent significant challenges to wind tunnel testing. Under tailwind conditions $\left(\psi=180^{\circ}\right)$ the ground vortex system comprises two symmetrically placed counter rotating vortices that are up to twice as strong as those formed under headwind conditions Out of all the arrangements investigated, the $\psi=180^{\circ}$ configuration generates the strongest vortex system. Under $150^{\circ}$ yaw conditions, the calculated flow-field comprises a complex system of ground and trailing vortices at high velocity ratios $\left(U^{*} \approx 20\right)$ 
which progressively simplifies to a single ground and trailing vortex as the velocity ratio is reduced.

The calculated behaviour of ground vortices formed under different yaw angles and intake velocity ratios has been considered in terms of vortex strength $\left(\Gamma^{*}\right)$ and fan-face coefficients:

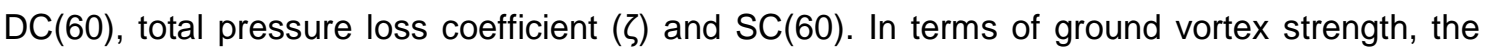
pure crosswind $\left(\psi=90^{\circ}\right)$ and pure tailwind $\left(\psi=180^{\circ}\right)$ cases are shown to be most critical. For the range of yaw angles, it is shown that there is a velocity ratio at which the vortex strength reaches a maximum. The trends also highlight, for this type of tightly wound vortex, the differences in the characteristics of the flow distortion descriptors based on swirl $\operatorname{SC}(60)$ and total pressure distributions $\mathrm{DC}(60)$.

\section{Acknowledgements}

The experimental work presented in this paper was conducted under an EPSRC studentship. The authors would like to thank Rolls-Royce for technical and financial support and in particular Chris Sheaf from Rolls-Royce Installations and Controls Engineering.

\section{References}

[1] Murphy J. Intake ground vortex aerodynamics [PhD thesis]. Cranfield: Cranfield University; 2008.

[2] Murphy J, MacManus DG. Intake ground vortex prediction methods. Journal of Aircraft 2011; 40(1): 23-33.

[3] Murphy J, MacManus DG, Sheaf C. Experimental investigation of intake ground vortices during takeoff. AIAA Journal 2010; 48(3): 688-701.

[4] Murphy J, MacManus DG. Ground vortex aerodynamics under crosswind conditions. Experiments in Fluids 2011; 50(1): 109-124. 
[5] Murphy J, MacManus DG. Ground vortex aerodynamics under headwind conditions. Aerospace Science and Technology 2011; In Press.

[6] Zantopp S, MacManus DG, Murphy J. Computational and experimental study of intake ground vortices. The Aeronautical Journal 2010; 114: Article 1162.

[7] Zantopp S. Jet engine ground vortex studies [MSc thesis]. Cranfield: Cranfield University; 2008.

[8] Rodert LA, Garrett FB. Ingestion of foreign objects into turbine engines by vortices. Washington: National Advisory Committee for Aeronautics; 1955 (Technical Report NACA TN 3330).

[9] Brix S, Neuwerth G, Jacob D. The inlet-vortex system of jet engines operating near the ground. AIAA Paper 2000; 2000(3998).

[10]De Siervi F, Viguier HC, Greitzer EM, Tan CS. Mechanisms of inlet vortex formation. Journal of Fluid Mechanics 1982; 124: 173-207.

[11] Shin HW, Greitzer EM, Cheng WK, Tan CS, Shippee CL. Circulation measurements and vortical structure in an inlet vortex flow field. Journal of Fluid Mechanics 1986; 162: 463487.

[12] Johns CJ. The aircraft engine inlet vortex problem. In AIAA Aircraft Technology, Integration and Operations 2002; 2002(5894).

[13] Seddon J, Goldsmith EL. Intake Aerodynamics: An Account of the Mechanics of Flow in and Around the Air intakes of Turbine-Engined and Ramjet Aircraft and Missiles. London: Collins; 1985.

[14] Motycka DL. Ground vortex-limit to engine/reverser operation. In Transactions of the ASME Gas Turbine Conference 1975; 75-GT-3. 
[15]Di Mare L, Simpson G, Sayma Al. Fan forced response due to ground vortex ingestion. In Proceedings of ASME Turbo Expo 2006: Power for Land, Sea and Air 2006; GT200690685.

[16] Green JS. Forced response of a large civil fan assembly. In Proceedings of ASME Turbo Expo 2008: Power for Land, Sea and Air 2008; GT2008-50319.

[17] Trapp LG, Girardi RM. Crosswind effects on engine inlets: the inlet vortex. Journal of Aircraft 2010; 47(2): 577-590.

[18] Nakayama A, Jones JR. Vortex formation in inlet flow near a wall. AIAA Paper 1996; 96(0803).

[19] Bissinger NC, Braun GW. On the inlet vortex system. National Aeronautics and Space Administration; 1974 (NASA CR-132536).,.

[20] Motycka DL, Walter WA, Muller GL. An experimental investigation of inlet ground vortex during reverse engine operation. AIAA Paper 1975; 75(1322).

[21] Motycka DL, Walter WA, Muller GL. An analytical and experimental study of inlet ground vortices. AIAA Paper 1973; 73(1313).

[22] Menter FR. Two-equation Eddy-viscosity turbulence models for engineering applications. AIAA Journal 1994; 32(8): 1598-1605.

[23] Ho WH, Jermy M. Validated CFD simulations of vortex formation in jet engine test cells. $16^{\text {th }}$ Australasian Fluid Mechanics Conference; 2007 December 3-7; Queensland.

[24] Karlsson A, Fuchs L. Time evolution of the vortex between an air inlet and the ground. AIAA Paper 2000; 2000(990).

[25]Roache P. Verification and Validation in Computational Science and Engineering. Albuquerque: Hermosa; 1998.

[26] Carmer C, Konrath R, Schroder A, Monnier J. Identification of vortex pairs in aircraft wakes from sectional velocity data. Experiments in Fluids 2008; 44: 367-380. 


\section{Accepted manuscript}

[27] Burley CL, Brooks TF, Van der Wall B, et al. Rotor Wake Vortex Definition - Initial Evaluation of 3-C PIV Results of the Hart-II Study. 28th European Rotorcraft Forum; 2002 September 17-20; Bristol UK. 
2011-12-08

\title{
Intake ground vortex characteristics
}

\author{
Mishra, N.
}

Sage

N Mishra, DG MacManus and JP Murphy, Intake ground vortex characteristics, Proceedings of the Institution of Mechanical Engineers, Part G: Journal of Aerospace Engineering. November 2012, vol. 226, no. 11, pp1387-1400

http://dx.doi.org/10.1177/0954410011424092

Downloaded from Cranfield Library Services E-Repository 